ESAIM : Proceedings, Vol. 7, 1999, 46-55

Third International Workshop on Vortex

Flows and Related Numerical Methods

http://www.emath.fr/proc/Vol.7/

\title{
Turbulent Flow Modeling Using a Fast, Parallel, Vortex Tube and Sheet Method
}

\author{
P. S. Bernard
}

\begin{abstract}
Department of Mechanical Engineering, University of Maryland, College Park, MD 20742. bernard@eng.umd.edu
\end{abstract}

\section{A. A. Dimas and J. P. Collins}

Krispin Technologies, Inc., 1370 Piccard Drive, Suite 210, Rockville, MD 20850.

adimas@krispintech.com, pcollins@krispintech.com

Vortex methods, particularly when regarded as a gridfree technique for large eddy simulation, represent a promising new alternative for modeling high Reynolds number, turbulent, engineering flows. To be successful, equal consideration must be given to efficiency and accuracy. Thus, the requisite number of vortex elements in the calculation should be kept to a minimum, though without sacrificing the essential flow physics. Moreover, it is necessary to incorporate a fast method for calculating the velocities due to many vortex elements from the Biot-Savart law, e.g. through use of a parallel implementation of the Fast Multipole method (Greengard and Rokhlin 1987). This paper describes our recent efforts in developing a vortex method with these capabilities. A brief discussion of some of the principal features of the method will be followed by a presentation of some preliminary results in modeling high Reynolds number flow past a 6:1 prolate spheroid.

\section{The vortex method}

At a minimum, our implementation of the vortex method needs to resolve the energy containing scales of turbulent flow down to, perhaps, the inertial range, as well as the dynamical processes leading to the generation of new coherent vortices near boundaries. At the same time, it is critical to prevent runaway growth in the number of vortex elements - a phenomenon which can occur when self-adaptive vortex methods attempt to resolve ever finer features of the turbulent field. A convenient means of accommodating these requirements is to make primary use of vortex tubes in representing the flow field. This opens up the possibility of using Chorin's hairpin removal and vortex reconnection scheme (Chorin 1993) as a natural sub-grid renormalization. In effect, the flow of energy to the dissipation range is stifled, and with it, unchecked production of vortex filaments, yet, hopefully, without harm to the natural cascade of energy into the inertial range.

Adjacent to solid boundaries, a smoothed vortex sheet structure is imposed for the purpose of efficiently capturing the wall normal vorticity flux - usually the only source of new vorticity entering the flow domain. The sheets - which encompass just a small number of parallel layers, typically 6 - 10 - have high aspect ratio in keeping with the large Reynolds number, so meaningful modeling of the thin viscous sublayer is possible.

The collection of sheets and tubes are advanced in time in approximation to the governing three- 
dimensional vorticity equation. For the filaments, this is accomplished according to the algorithm:

$$
\underbrace{\frac{\partial \boldsymbol{\Omega}}{\partial t}+(\nabla \boldsymbol{\Omega}) \mathbf{u}=(\nabla \mathbf{u}) \boldsymbol{\Omega}}_{\text {convect filament endpoints }}+\underbrace{\frac{1}{R_{e}} \nabla^{2} \boldsymbol{\Omega}}_{\text {decay model }},
$$

where $R_{e}$ is the Reynolds number. As the tubes lengthen, they are divided into smaller segments. The decay model, describe in the next section, is a way of modeling long time diffusive effects on the vortex tubes. In particular, we may assume that viscous dissipation plays some role in the demise of coherent vortices in a turbulent flow. This may be of some concern in internal flows, such as periodic channel flow, where vortices, once present, will generally persist in the calculation for long periods. In contrast, it is less of a priority in external flows where the viscous decay of vortices can be assumed to take place downstream of the region of interest. Apart from this aspect of the algorithm, (1) is a standard approach for filament calculations (Chorin 1993).

The vortex sheets are advanced through time according to the prescription:

$$
\underbrace{\frac{\partial \boldsymbol{\Omega}}{\partial t}+(\nabla \boldsymbol{\Omega}) \mathbf{u}}_{\text {convect sheets \& interpolate }}=\underbrace{(\nabla \mathbf{u}) \boldsymbol{\Omega}+\frac{1}{R_{e}} \nabla^{2} \boldsymbol{\Omega}}_{\text {finite dif ferences }} .
$$

In this, convection of the sheets is immediately followed by their interpolation back into the mesh as part of a fractional step method. The end result is to have the sheet vorticity field available on the uniform mesh during every time step so that finite differences can be efficiently used to estimate the wall-normal diffusion term. For the calculations described below, the stretching term is approximated via

$$
(\nabla \mathbf{u}) \boldsymbol{\Omega}=|\boldsymbol{\Omega}| \frac{\partial \mathbf{u}}{\partial s}=|\boldsymbol{\Omega}| \frac{(\mathbf{u}(\mathbf{x}+\mathbf{s} \Delta r / 2)-\mathbf{u}(\mathbf{x}-\mathbf{s} \Delta r / 2))}{\Delta r}
$$

where $s$ is a coordinate in the direction of the vorticity vector and $\mathbf{s}$ is a unit vector in that direction. Use of (3) removes the need to develop elaborate differencing formulas for the prolate spheroid geometry. Since only wall normal diffusion is of interest, it is a simple matter to calculate diffusion via finite differences. It should be remarked that the use of (2) and (3) is provisional. New computations will use an unstructured triangularization of the surface sheet mesh and associated finite volume formulas in representing the convection, stretching and diffusion terms in (2). In addition, the vorticity in the triangularization is piecewise linear, while earlier computations on the rectangular mesh used piecewise constant vorticity.

\section{Vortex creation and destruction}

Two different models of the vortex destruction process have been developed in order to help restrict the vortices in the calculation to those which are most dynamically important. The intent is also to mimic the reality that coherent vortices in turbulent flow do have a finite lifespan. Future research will look into optimal ways of accommodating this process, though it should be noted, as suggested above, that these considerations are of minimal concern for the case of the prolate spheroid flow.

Preliminary calculations of channel flow using this method have shown that vortices which have been in the computation for long periods often acquire many segments, and tend to be without 
favored orientation. This suggests the practice of removing such structures when they appear. An alternative approach is to consider the accumulated effect of vortex stretching versus viscous spreading on each individual tube. The well known Gaussian core solution (Leonard 1975) establishes an equilibrium radius for tubes, $r_{e}$, in which the effects of vortex stretching are in equilibrium with diffusion. For a tube whose length increases from $\Delta s$ to $\Delta s^{\prime}$ in time $\Delta t$ it may be shown that

$$
r_{e}=2 \sqrt{\frac{\Delta t}{R_{e}\left(\Delta s^{\prime} / \Delta s-1\right)}}
$$

Generally, the radius of a vortex is either above or below the $r_{e}$ value associated with the local stretching experienced by the vortex at the given Reynolds number. If $r>r_{e}$, the vortex tends to get thinner, if $r<r_{e}$, the vortex tends to widen. While it may be possible to model the process by which vortices change core size (Rossi 1995), such an approach is unsuited to the present purposes, since it fails to provide for retiring of old structures. The latter objective can be fulfilled, however, by providing for a loss of circulation from the tubes whenever $r<r_{e}$. The amount is determined by the extent to which vorticity would diffuse beyond $r$ due to the imbalance of vortex stretching and diffusion. The necessary relation is

$$
\Gamma^{n+1}=\Gamma^{n}\left(1-\frac{4 \Delta t}{R_{e}}\left(\frac{1}{r^{2}}-\frac{1}{r_{e}^{2}}\right)\right)
$$

where $\Gamma^{n}$ is the circulation at time $n$. If $r>r_{e}$, the circulation may be left the same, i.e.

$$
\Gamma^{n+1}=\Gamma^{n}
$$

and if the tube faces a net contraction, i.e. $\Delta s^{\prime}<\Delta s$, then the maximum circulation loss can be assessed, namely

$$
\Gamma^{n+1}=\Gamma^{n}\left(1-\frac{4 \Delta t}{R_{e}} \frac{1}{r^{2}}\right)
$$

For tubes composed of multiple segments, the average of $\Delta s$ and $\Delta s^{\prime}$ over all segments can be used.

As the scheme is presently constituted, all vortices have the identical radius. Since high shear near the walls leads to high stretching rates and hence small $r_{e}$, the influence of (5) or (7) in reducing $\Gamma$ should be most pronounced away from the wall where vortices are less organized and experience slower rates of stretching. Whenever the circulation of a vortex drops below a threshold, it is dropped from the calculation, thus providing a second means for eliminating vortices.

Providing for each new generation of vortices as they are produced in a turbulent flow is also a critical aspect of the numerical scheme. The algorithm must at the same time be both sensitive to the physical process by which new structures appear, yet not so unconstrained as to allow for the formation of impossibly large numbers of new vortices. A successful means of accommodating these conditions has been previously developed (Bernard 1996, 1998). In this, whenever vorticity initially produced at the boundary in fulfillment of the no-slip condition is ejected in sufficient strength beyond the sheet domain, it is formed into new tubes. In the turbulent boundary layer some of the vortices subsequently stretch and reorient to become a new generation of structures capable of causing the creation of new vortices. Large numbers of new vortex tubes are also associated with flow separation as occurs in the prolate spheroid flow. 


\section{Velocity field computation}

Following standard practice (Puckett 1993), the velocity field is computed as a numerical approximation to

$$
\mathbf{u}(\mathbf{x}, t)=\int_{\Re^{3}} K_{\eta}\left(\mathbf{x}-\mathbf{x}^{\prime}\right) \boldsymbol{\Omega}\left(\mathbf{x}^{\prime}, t\right) d \mathbf{x}^{\prime}
$$

where $K_{\eta}$ is the smoothed Biot-Savart kernel. In the case of sheets,

$$
K_{\eta}= \begin{cases}K & |\mathbf{x}| \geq \eta \\ K\left(\frac{5}{2}-\frac{3}{2}\left(\frac{\mathbf{x}}{\eta}\right)^{2}\right) \frac{|\mathbf{x}|^{3}}{\eta^{3}} & |\mathbf{x}|<\eta\end{cases}
$$

where

$$
K(x, y, z)=-\frac{1}{4 \pi|\mathbf{x}|^{3}}\left(\begin{array}{ccc}
0 & -z & y \\
z & 0 & -x \\
-y & x & 0
\end{array}\right),
$$

and (8) may be simplified to

$$
\int_{\Re^{3}} K_{\eta}\left(\mathbf{x}-\mathbf{x}^{\prime}\right) \boldsymbol{\Omega}\left(\mathbf{x}^{\prime}, t\right) d \mathbf{x}^{\prime}=\mathbf{A}\left(\mathbf{x}-\mathbf{x}_{i}\right) \times \boldsymbol{\Omega}_{i}
$$

if it is assumed that the vorticity is constant over the sheets. In the far field for a rectangular Cartesian coordinate system

$$
\begin{aligned}
& A_{1}=-\frac{h_{j}}{8 \pi} \ln \left(\frac{r_{11}-Z_{1}}{r_{11}+Z_{1}} \frac{r_{21}+Z_{1}}{r_{21}-Z_{1}} \frac{r_{22}-Z_{2}}{r_{22}+Z_{2}} \frac{r_{12}+Z_{2}}{r_{12}-Z_{2}}\right) \\
& A_{2}=-\frac{h_{j}}{4 \pi}\left(\tan ^{-1} \frac{X_{2} Z_{2}}{Y r_{22}}-\tan ^{-1} \frac{X_{2} Z_{1}}{Y r_{21}}-\tan ^{-1} \frac{X_{1} Z_{2}}{Y r_{12}}+\tan ^{-1} \frac{X_{1} Z_{1}}{Y r_{11}}\right) \\
& A_{3}=-\frac{h_{j}}{8 \pi} \ln \left(\frac{r_{11}-X_{1}}{r_{11}+X_{1}} \frac{r_{21}+X_{1}}{r_{21}-X_{1}} \frac{r_{22}-X_{2}}{r_{22}+X_{2}} \frac{r_{12}+X_{2}}{r_{12}-X_{2}}\right)
\end{aligned}
$$

where $r_{m n}^{2}=X_{m}^{2}+Y^{2}+Z_{n}^{2}, m, n=1,2, X_{1}=\left(x_{i}-x_{j}-l_{j}\right) / \eta, X_{2}=\left(x_{i}-x_{j}+l_{j}\right) / \eta, Z_{1}=$ $\left(z_{i}-z_{j}-d_{j}\right) / \eta, Z_{2}=\left(z_{i}-z_{j}+d_{j}\right) / \eta$, and $Y=\left(y_{i}-y_{j}\right) / \eta$. Within the distance $\eta$ of the sheets, local versions of these formulas may be obtained analytically.

In the case of tubes, the common approximation

$$
\int_{\Re^{3}} K_{\eta}\left(\mathbf{x}-\mathbf{x}^{\prime}\right) \boldsymbol{\Omega}\left(\mathbf{x}^{\prime}, t\right) d \mathbf{x}^{\prime}=-\frac{\Gamma_{i}}{4 \pi} \frac{\mathbf{r}_{i} \times \mathbf{s}_{i}}{\left|\mathbf{r}_{i}\right|^{3}} \phi(r / \sigma)
$$

may be made where

$$
\phi(r)=\left(1-\left(1-\frac{3}{2} r^{3}\right)\right) e^{-r^{3}}
$$

is a high-order smoothing function (Chorin, 1993). Additionally, when the velocity due to a sheet at $x_{i}$ is sought at points $\mathbf{x}$ satisfying $\left|\mathbf{x}-\mathbf{x}_{i}\right| \gtrsim .5,(10)$ and (11) are fully equivalent if the sheet is viewed as being a tube with

$$
\mathrm{s}=\Delta s \frac{\Omega}{|\Omega|}
$$

and

$$
\Gamma \Delta s=|\boldsymbol{\Omega}| V_{s}
$$


where $V_{s}$ is the volume of the sheet and $\Delta s$ is arbitrary. The relative simplicity of the tube formulas in comparison to (10) yields a significant gain in efficiency in computing these far field interactions. Moreover, in a fast multipole algorithm, such as is implemented here (Dimas et al. 1998), letting sheets produce a velocity field as if they were tubes enables them to participate in the fast calculation.

In summary, the velocity is computed from

$$
\mathbf{u}(\mathbf{x}, t)=\sum_{i=1}^{N_{\text {sheets }}} \mathbf{A}\left(\mathbf{x}-\mathbf{x}_{i}\right) \times \boldsymbol{\Omega}_{i}-\frac{1}{4 \pi} \sum_{i=1}^{N_{\text {segments }}} \frac{\mathbf{r}_{i} \times \mathbf{s}_{i}}{\left|\mathbf{r}_{i}\right|^{3}} \Gamma_{i} \phi(r / \sigma)
$$

together with a potential flow that forces the non-penetration condition to be satisfied at solid boundaries. The latter field is readily computed from standard techniques using boundary source elements at the locations of the wall sheets (Hess and Smith 1967).

\section{Prolate spheroid flow}

The flow past a 6:1 prolate spheroid under a variety of conditions has been the object of considerable attention in physical experiments (Chesnakas and Simpson 1994, Wetzel et al. 1998) and thus provides a convenient venue with which to examine the effectiveness of our vortex method in predicting high Reynolds number flows. In the case studied here, the Reynolds number based on free stream velocity and axial length of the spheroid is $R_{e}=4,200,000$. Results have been obtained for $0^{\circ}, 20^{\circ}$ and $30^{\circ}$ angle of attack. In these, the surface mesh is $64 \times 6 \times 16$ in the axial, surface normal and azimuthal directions, respectively. The sheets have thickness .0015 and aspect ratio on the order of 20 . The sheet thickness is just slightly larger than that necessary to well resolve the viscous sublayer at the Reynolds number of interest. Further reduction in the sheet thickness will be possible when triangular sheet elements are used, since they better resolve the curvature of the spheroid than the current rectangular elements.

A limit on the time step is imposed by the requirement that sheets do not convect beyond their neighbor's position. The nominal value of $\Delta t=0.0025$ accomplishes this; only rarely in the computations shown here must $\Delta t$ be reduced to satisfy this condition. The present calculations have been run for approximately 1000 time steps covering an elapsed time of $t=2.5$. This covers primarily the startup flow plus what appears to be part of the first period of a cyclically evolving wake.

Though still preliminary, the computed results are encouraging in a number of important aspects. In particular, the computational algorithm is fully stable, and is not subject to run-away growth in the number of vortex elements. Rather, an initial rapid growth in the number of vortices becomes essentially linear in time, a rate which is compatible with the continuous development of the wake. We may conclude that the vortex destruction, production and hairpin removal algorithms are working as anticipated.

It is also interesting to observe the time history of the number of sites producing vortices shown in Fig. 1. The maximum of this is 1024 corresponding to the number of surface sheets. It is seen that during the time period $0.6 \leq t \leq 1.0$ virtually the entire surface of the spheroid is encased in new vortex tubes as a final response to the impulsive start of the spheroid. Subsequently, as the turbulent wake is formed, the region producing new vortices shrinks to approximately half the 
surface. For the $20^{\circ}$ case this is primarily on the leeward side where significant flow separation is occurring. The windward side experiences a compression of the boundary layer that works against the appearance of new tubes. It should also be noted that the underlying sheet vorticity is generally non-steady in character regardless of whether the conditions under which new tubes appear are satisfied. Finally, the figure shows that at $0^{\circ}$ angle of attack a greater part of the surface is producing vortices than the $20^{\circ}$ case, though clearly a final equilibrium has yet to be reached.

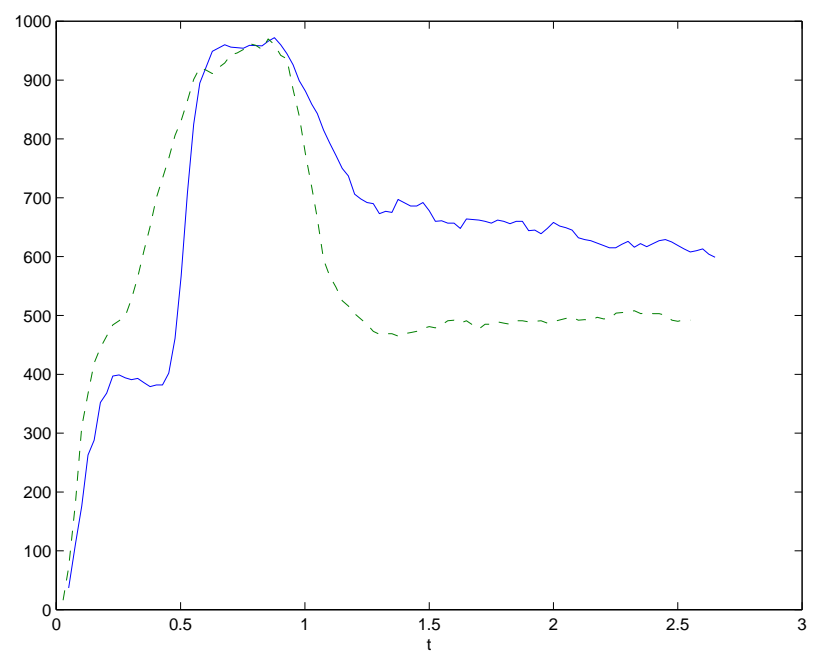

Figure 1: Number of cites producing new tubes. $-0^{\circ}$ angle of attack; $---20^{\circ}$ angle of attack.

The vortex filament distribution at $t=2.5$ as viewed from the side and top of the $20^{\circ}$ angle of attack case are given in Figs. 2 and 3, and a similar side view of the $30^{\circ}$ case is in Fig. 4.

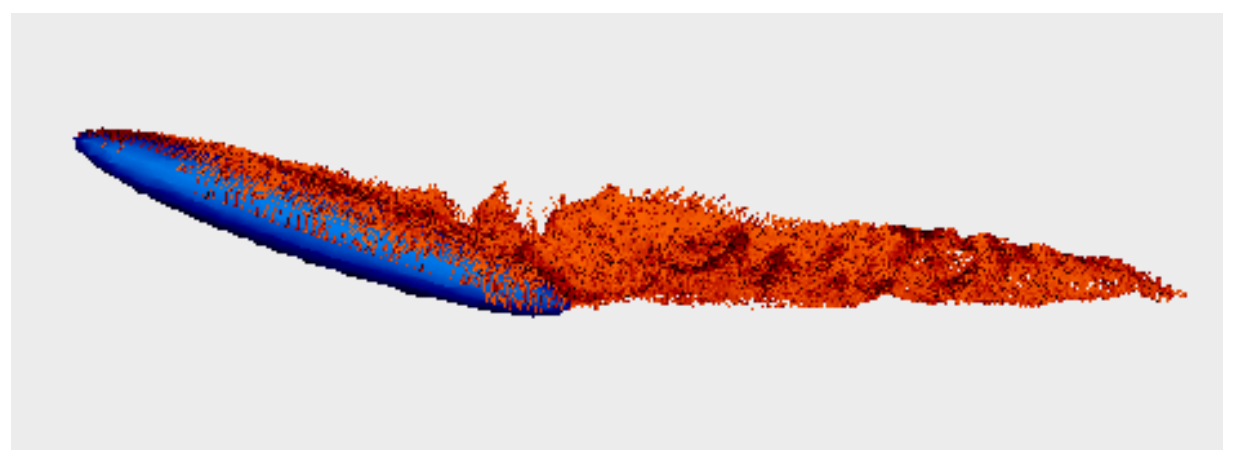

Figure 2: Prolate spheroid flow, $20^{\circ}$ angle of attack, side view at $t=2.5$.

At the high Reynolds number in these calculations the wake is relatively narrow. This illustrates one of the great strengths of the vortex method: the computational effort is confined to just the support of the vorticity field, which is clearly a relatively small part of the whole flow domain. It also may be observed how the computed vortex system, which consists of approximately 90,000 vortex segments at $t=2.5$, has naturally formed into a series of larger scale coherent vortical motions which give a definite structure to the wake.

It is seen in the figures that vortex tube generation is primarily on the back surface of the spheroid. The flow is clearly fully turbulent with unsteady separation occurring towards the rear. Figure 5 gives a closer look at the vortical structure in the separation zone near the rear end. Plotted here 


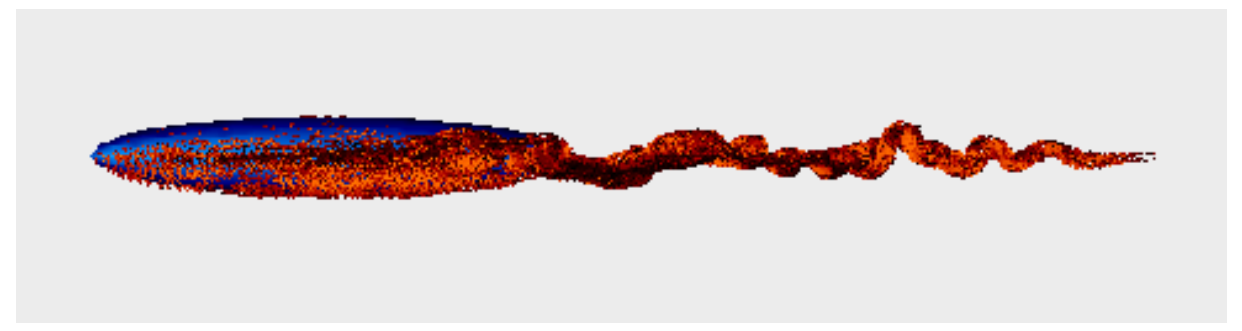

Figure 3: Prolate spheroid flow, $20^{\circ}$ angle of attack, top view at $t=2.5$.

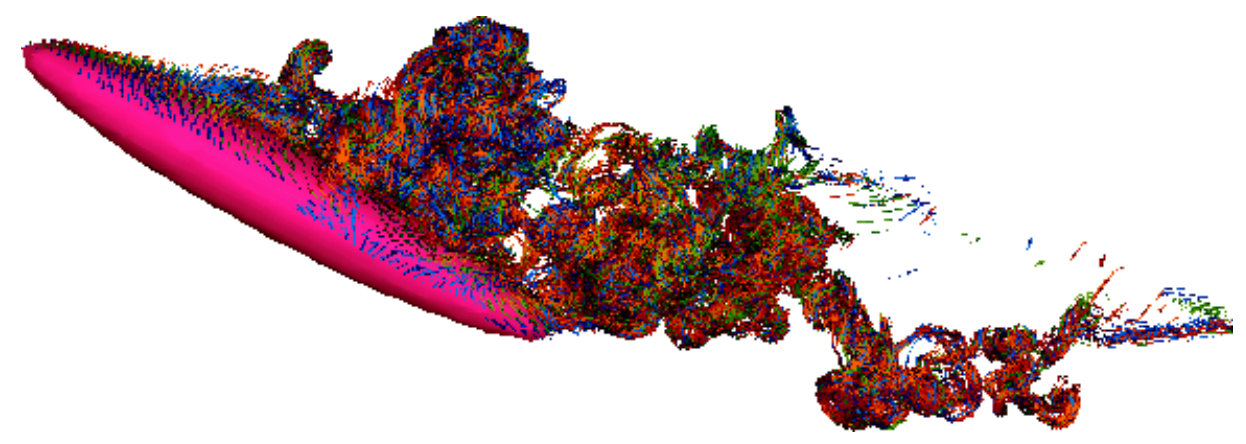

Figure 4: Prolate spheroid flow, $30^{\circ}$ angle of attack, side view at $t=2.5$.

are just vortices whose strengths are in the top $20 \%$ of all vortices. Clearly, this region contains several significant coherent vortices engaged in a complex swirling motion as they lift up off the surface. Such motions continue on into the wake as shown in Figs. $2-4$.

Examination of the time evolution of the flow at $20^{\circ}$ angle of attack reveals how the flow evolves into the turbulent field shown in Figs. 2 and 3. The process unfolds first with the appearance of counter-rotating axial vortices on the leeward side of the prolate spheroid. These are formed as a result of the oblique flow over the surface converging toward the central axis. In short order a spanwise oscillation appears in the vortex pair, which grows in amplitude until the vortices interact forming a larger, swirling structure. Rapid development of the turbulent wake follows as the vortex convects off the body with new vortices developing behind.

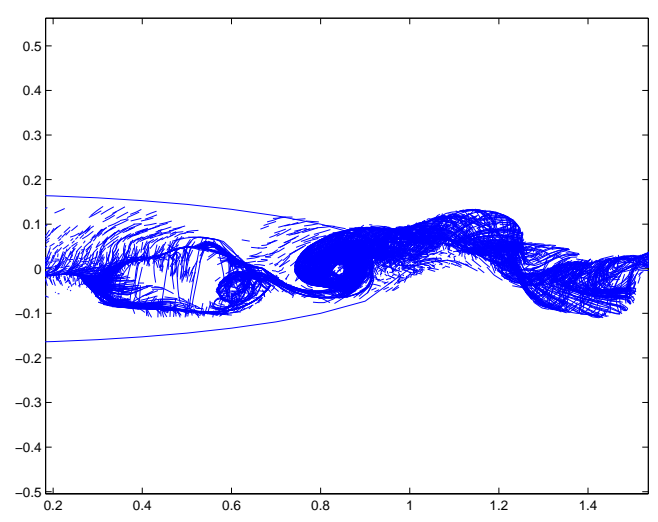

Figure 5: Close up of prolate spheroid flow at rear edge, $20^{\circ}$ angle of attack, top view at $t=2.5$.

Other attractive features of the solutions include sharp separation off the rear tip and persistence of the wake vortices in time. This highlights an advantage of the non-diffusive property of vortex 
methods, since typical RANS or LES calculations tend to prematurely dissipate shed vortices.

A plot of the surface pressure contours for the $0^{\circ}$ and $20^{\circ}$ flows is given in Fig. 6. The abscissa here is the index of the mesh in the azimuthal direction running from 1 to 16 , while the ordinate is axial position along the body running from 1 to 64 . Indices 1 and 16 in the azimuthal direction are at the center of the leeward side of the spheroid, while 8 and 9 are at the center of the windward side. The pressure was computed using a numerical solution to the integral pressure formulation developed by Uhlman (1992).
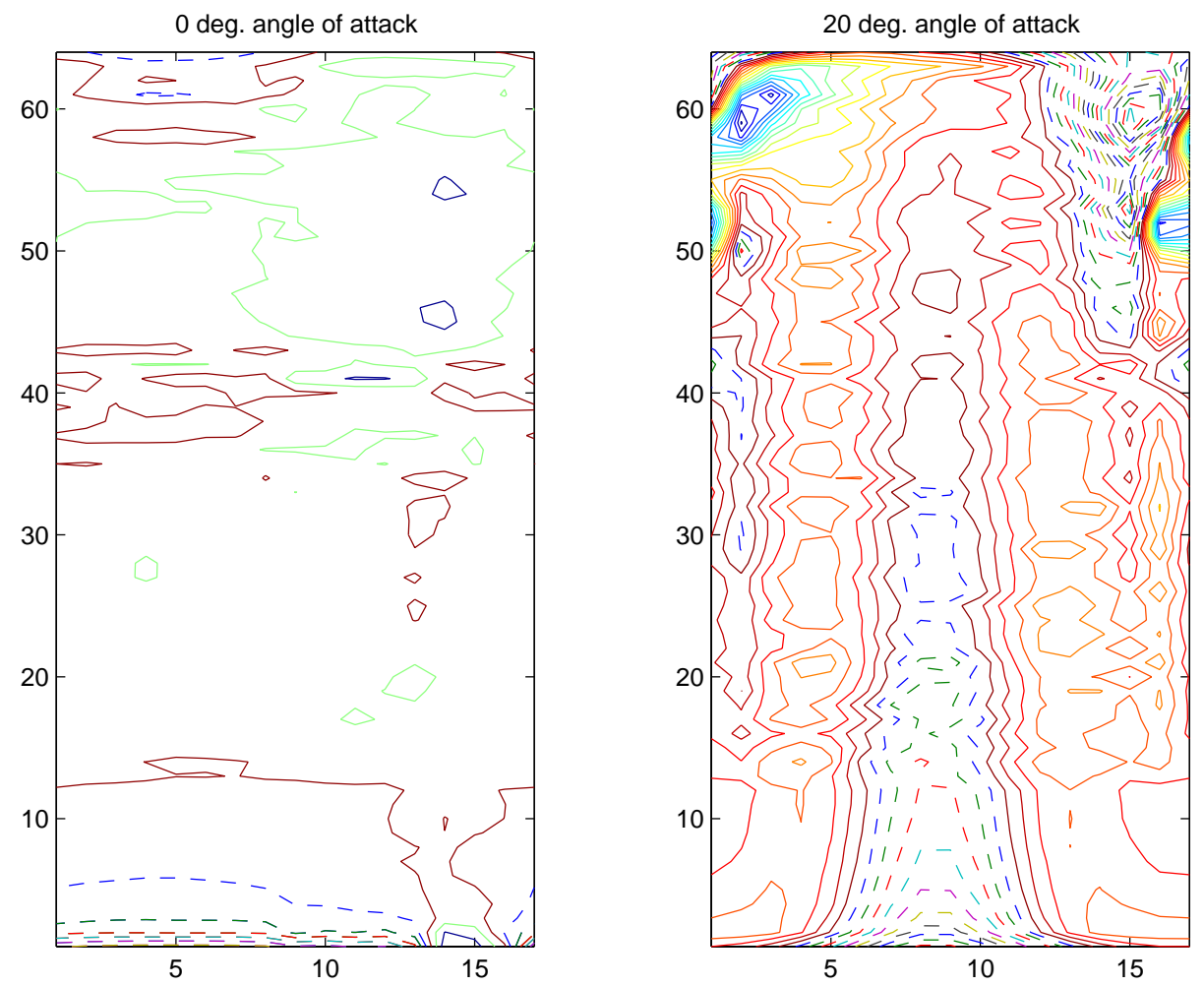

Figure 6: Pressure contours. —— negative pressure; --- positive pressure.

The contrast in contours between the two cases displayed in Fig. 6 reflects the difference between the massive asymmetrical flow separation of the $20^{\circ}$ degree case vs. the essentially attached flow of the $0^{\circ}$ degree case. The pressure solver successfully captures these distinctions, e.g. note the large high pressure region on the leeward side of the $20^{\circ}$ case. The effect of individual wake structures on the pressure field is evident in the large positive/negative pressure variations in the rear one third of the leeward side of the $20^{\circ}$ simulation. Note, as well, that the pressure near the front end of the $0^{\circ}$ case implies that the incoming flow is slightly at an angle caused by asymmetrical positions of downstream structures. A direct comparison of computed pressures with experimental data for the $30^{\circ}$ case is shown in Fig. 7. The abscissa runs from $0^{\circ}$ degrees at the windward side to $180^{\circ}$ at the leeward side. The top graph is at a point just beyond the mid section while the lower one is near the end of the body.

The force history on the prolate spheroid for the $0^{\circ}$ and $20^{\circ}$ cases is shown in Fig. 8 . For the $0^{\circ}$ angle of attack case, the drag, lift and side force coefficients are shown, while for the $20^{\circ}$ case the drag and lift coefficients are given. The non-steadiness of the forces reflects the unsteady shedding of vortical structures into the wake. Also included in the figure (as straight lines) are the average 
drag and lift during the time period $1.5 \leq t \leq 2.5$ which gives some idea of the overall force predictions. The mean drag values are approximately .06 and .1 for the $0^{\circ}$ and $20^{\circ}$ angle of attack cases, respectively, while the mean lift is approximately .23 for the $20^{\circ}$ case. The mean lift and side force should be zero for the case with no angle of attack. It is evident from the figure that the averaging period available to this run is insufficient to see this trend. For example, a quite significant side force is felt which is just beginning to dissipate at the end of the calculation. We assume that the side force will oscillate indefinitely along with the lift and that this mimics a real physical effect.
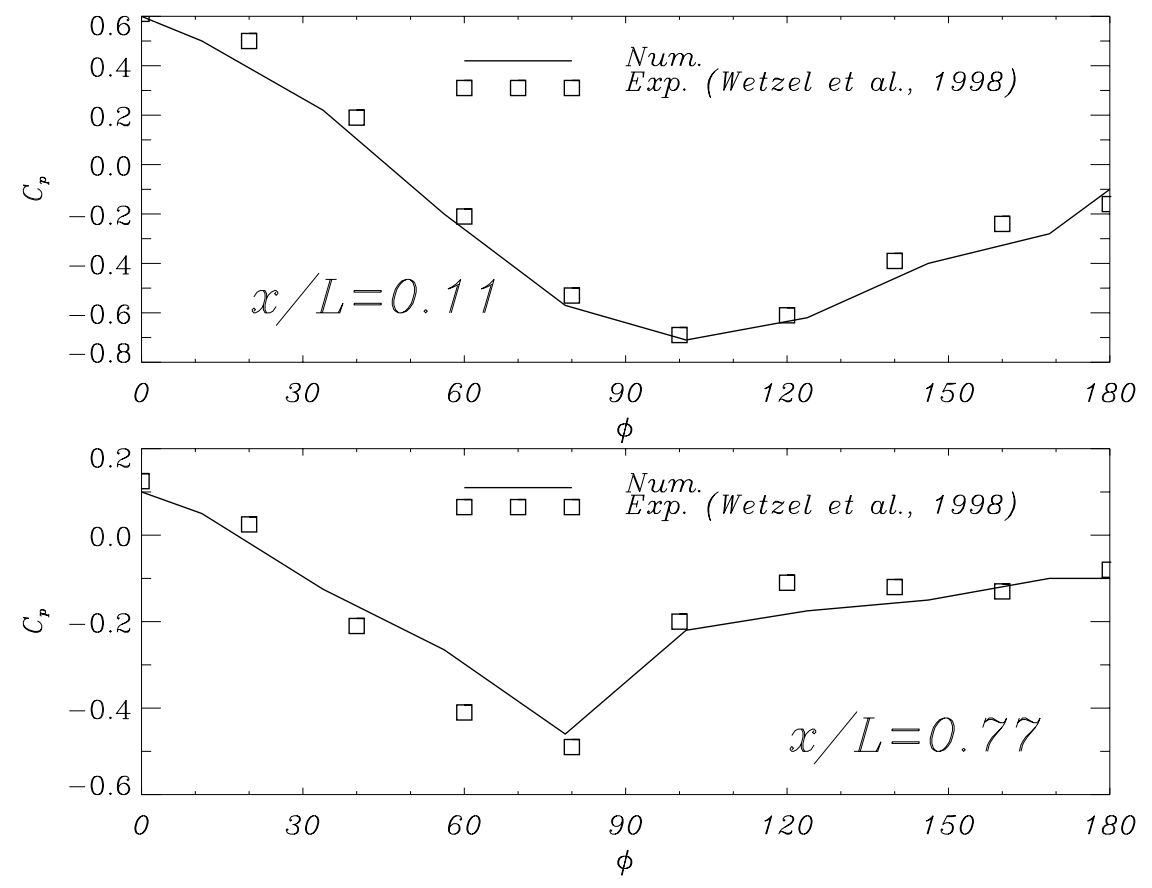

Figure 7: Comparison of mean pressures with experiment for flow at $30^{\circ}$ angle of attack.

\section{Conclusion}

The vortex method for turbulent flow simulation described herein has made a promising start toward achieving the capability for efficiently modeling physically realistic turbulent flows. Reasonable force and pressure predictions have been made; flow and wake structure developing on the prolate spheroid show many realistic features. Further work will investigate the sensitivity of the approach to the various simplifications used in creating an efficient scheme, and attempt to demonstrate a close connection to experimental predictions over a range of different flows.

Acknowledgment We wish to thank Dr. John Grant for help in the development of the potential flow and pressure solvers used in this code. Funding for this work has been provided by the U. S. Dept. of Energy through SBIR Grant DE-FG02-97ER82413.A001.

\section{References}

1 Bernard, P. S.: A vortex method for wall bounded turbulent flows. Vortex Flows and Related Numerical Methods, II, edited by Y. Gagnon et al., http://www.emath.fr/proc/Vol.1/, ESAIM: Proc., (1996) 15-31. 

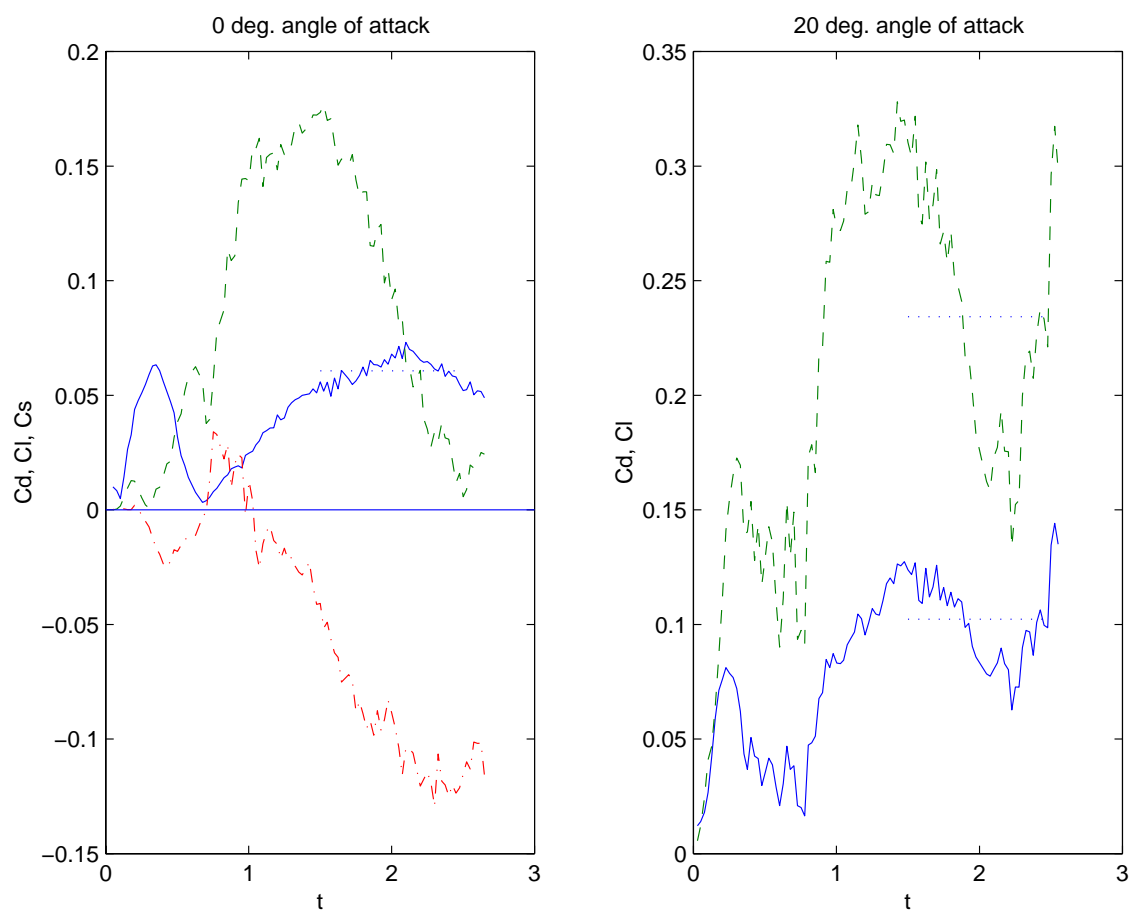

Figure 8: Force coefficient histories.

2 Bernard, P. S.: Toward a vortex method simulation of non-equilibrium turbulent flows. To appear in Proc. ICASE/LaRC/AFOSR Symposium on Modeling Complex Turbulent Flows, Kluwer Pub. Inc. (1998)

3 Chesnakas, C. J., And Simpson, R. L.: Full three-dimensional measurements of the cross-flow separation region of a 6:1 prolate spheroid. Experiments in Fluids 17 (1994) 68-74.

4 Chorin, A. J.: Hairpin removal in vortex interactions II. J. Comput. Phys. 107 (1993), 1 - 9.

5 Dimas, A., Collins, J. P. And Bernard, P. S.: A fast, parallel vortex method for turbulent flow simulation. Proc. ASME Fluids Engineering Div. Summer Mtg. (1998).

6 Greengard, L. and Rokhlin, V. : A fast algorithm for particle simulations. J. Comput. Phys. 73 (1987), $325-348$.

7 Hess, J. L. And Smith, A. M. O.: Calculation of potential flow about arbitrary bodies. Prog. Aero. Sci. 8 (1967), 1 - 138.

8 LeOnARD, A.: Numerical simulation of interacting, three-dimensional vortex filaments. Lec. Notes in Phys., 35 (1975), 245 - 250.

9 PUCKETT, E. G.: Vortex methods: an introduction and survey of selected research topics. In Incompressible computational fluid dynamics: trends and advances, edited by M. D. Gunzburger and R. A. Nicolaides, Cambridge University Press, Cambridge, (1993) 335-407.

10 Rossi, L.: Resurrecting core spreading vortex methods: a new scheme that is both deterministic and convergent. SIAM J. Sci. Comp. 17 (1995), 370.

11 Uhlman JR., J. S.: An integral equation formulation of the equations of motion of an incompressible fluid. NUWC-NPT Technical Report 10,086, Naval Undersea Warfare Center Division, Newport, Rhode Island (1992).

12 Wetzel, T. G., Simpson, R. L., and Chesnakas, C. J.: Measurement of three- dimensional crossflow separation. AIAA Journal 36 (1998) 557-564. 